\title{
A Methodology for Choosing between Route Deviation and Point Deviation Policies for Flexible Transit Services
}

\author{
Yue Zheng $\mathbb{D}^{1},{ }^{1}$ Wenquan Li $\mathbb{D},{ }^{1}$ and Feng Qiu $\mathbb{D}^{2}$ \\ ${ }^{1}$ Jiangsu Key Laboratory of Urban ITS, Jiangsu Province Collaborative Innovation Center of Modern Urban Traffic Technologies, \\ School of Transportation, Southeast University, Nanjing, China \\ ${ }^{2}$ Department of Computer Science, University of Victoria, Victoria, Canada
}

Correspondence should be addressed to Wenquan Li; wenqli@seu.edu.cn

Received 3 February 2018; Revised 30 June 2018; Accepted 30 July 2018; Published 12 August 2018

Academic Editor: Antonino Vitetta

Copyright (C) 2018 Yue Zheng et al. This is an open access article distributed under the Creative Commons Attribution License, which permits unrestricted use, distribution, and reproduction in any medium, provided the original work is properly cited.

\begin{abstract}
Flexible transit services, which bring together the characteristics of fixed-route transit and demand-responsive transit, have been proven to be cost-efficient in low-density residential areas. In this paper, a methodology is proposed to assist planners in making better decisions when choosing between route deviation policy and point deviation policy, which are two promising types of flexible transit services. A user cost function is developed to measure the service quality of the transit systems, and analytical models are constructed to compare the system performance under both expected and unexpected demand levels. Based on the experiments for various scenarios over a real-life transit example, the critical demands, which represent the switching point between the two competing service policies, have been derived. Our findings show that point deviation policy is more efficient at low-demand levels, while route deviation policy is a better choice at low-to-moderate demand levels. At unexpectedly high demand levels, route deviation policy is better able to accommodate rejected passengers than point deviation policy.
\end{abstract}

\section{Introduction}

With the acceleration of urbanization in recent decades, an increasing number of suburban areas with low population density have arisen. The increasing dispersion of population results in a low degree of resource sharing for traditional fixed-route transit, which has a rigid route and schedule structure. Demand-responsive transit, which can provide much of the desired flexibility with curb-to-curb services, is considered costly to deploy and is therefore mostly limited to specialized operations such as paratransit for people with reduced mobility [1].

To address these issues, researchers and practitioners seek to introduce flexible transit services to meet the needs of new travel patterns in suburban and rural areas. All these flexible transit systems combine the efficiency of fixed-route transit with the flexibility of demand-responsive transit. They are regarded as fixed-route services in that they have a set of stops with predetermined schedules. On the other hand, they are considered as demand-responsive systems because passengers can ask for service at optional locations. Previous works based on actual operational data have shown that flexible transit services are more cost-efficient than pure demand-responsive transit [2] and conventional bus systems [3] in low-demand areas.

Based on the investigation by Koffman [4], flexible operating policies can be categorized into six main types: route deviation, point deviation, demand-responsible connector (DRC), request stops, flexible route segments, and zone route. Among these flexible services, DRC and zone route are implemented as feeder lines that have only one transfer point. Request stops can be regarded as a route deviation where only a few deviations are available. Flexible route segments operate in point deviation mode in some portion of the route. Hence, a comparison between route deviation and point deviation can generally reflect the difference in system structure for non-feeder services.

According to a survey by Potts et al. [5], nearly $40 \%$ of transit agencies in the United States and Canada operate flexible transit services. Among these, route deviation is by far the most common form (up to $63.9 \%$ ), while point deviation only accounts for $16 \%$. One of the main factors 
hindering the widespread use of point deviation service is the sophisticated support systems required to schedule the curb-to-curb requests [6]. However, with the rapid development of intelligent transportation systems, point deviation is becoming a promising operating policy from a long-term perspective.

Most of these studies revealed that flexible transit services are promising operating policies for shaping new travel patterns in low-demand areas and passengers are generally willing to use these innovative transit systems [7, 8]. However, how to choose the most appropriate policy in a certain service area, especially between route deviation policy and point deviation policy, remains a challenge for transit operators.

In this paper, we present a methodology to assist decision makers in choosing between route deviation and point deviation policies. Analytical models are developed to evaluate the system performance of two competing services. Using the analytical models, the critical demand, in which the two transit services have the same performance, can be identified, and the reliabilities of the two systems are further discussed. Our work serves as a meaningful step towards selecting between flexible transit services in areas with low and fluctuating travel demand.

\section{Literature Review}

Route deviation and point deviation policies differ in terms of degree of flexibility. More specifically, the route deviation systems are much more constrained than those allowed for point deviation; they must operate along a well-defined path and deviate to serve curb-to-curb requests within a service area around the path [9]. On the other hand, route deviation systems have a lower administrative/dispatch requirements and are easier to operate than point deviation services. Some transit agencies (for example, Tennessee Department of Transportation [10]) find it is a hard to choose between route deviation and point deviation policies when deploying new flexible transit services.

Although route deviation has been the most popular type of flexible transit services [4], it has been seldom studied by researchers. Most existing studies focused on improving the planning and scheduling of point deviation services. Quadrifoglio et al. [11-13] evaluated the upper and lower bounds of the maximum longitudinal velocity of service vehicles and proposed scheduling algorithms of point deviation service for both static and dynamic scenarios. Fu [14] developed a model to reveal the relationship between feasible deviations, slack time, zone size, and dwell time. Zhao and Dessouky [15] analyzed the relationship between the service cycle time and the length and width of the service area. Nourbakhsh and Ouyang [16] developed a new structured point deviation system that outperforms fixed-route transit under low-tomoderate demand levels. Qiu et al. [17] explored the feasibility of replacing fixed-route transit with point deviation policies and derived the switching demand densities between the two policies. Qiu et al. [18] presented a new demi-flexible transit system and compared its performance with fixedroute and point deviation services. Yang et al. [19] proposed a GIS-based method to select the most cost-effective route for deviated fixed-route transit (DFRT) based on the lowest operating cost per passenger. Chen and Nie [20] developed a new hybrid system that integrates the fixed-route service with point deviation service. The point deviation system serves as a connector between passengers' origin/destination and the fixed-route service to improve accessibility.

Most previous studies are conducted in operating environments with expected and predictable demand. Only a very limited number of studies focus on the uncertainty of travel demand in low-demand areas. Qiu et al. [21] proposed a dynamic station strategy to accept more passengers when the actual travel demand is higher than demand. Zheng et al. [22] proposed a slack arrival strategy to reduce the number of rejected passengers through redistributing the slack time among segments. In practice, the actual demand frequently deviates from the expected demand levels in low-demand service areas and the uncertain travel demand makes it hard to serve all curb-to-curb requests, which definitely degrade the service level of the systems.

In this paper, we aim to investigate and establish the conditions that are fit for the implementation of route deviation or point deviation policy. The system performances are compared not only at expected demand levels, but also at unexpected demand levels to test the reliability of the two transit services. To our knowledge, this paper is the first to develop a methodology for solving this problem.

\section{System Description}

3.1. Service Areas and Demand. The service area in our study represents a residential community and is modeled as a rectangle of width $W$ and length $L$, delimited by two terminal checkpoints located at connection centers (see Figures 1 and 2 ). The transit system serves as community buses to transport passengers between their houses and connection centers, where they can transfer to the major transit network.

In general, the number of transit lines passing by the connection centers is very high; therefore, the temporal demand distribution at the connection centers is assumed to follow a Poisson distribution. It is also reasonable to assume that the trip origins and destinations outside checkpoints are uniformly and independently distributed in the service area according to a homogeneous spatial Poisson process. There are three main types of passengers in the service area with proportions of $\gamma_{1}, \gamma_{2}$, and $\gamma_{3}$ defined as follows $\left(\gamma_{1}+\gamma_{2}+\gamma_{3}=\right.$ 1).

Type I: pick up and drop off both at checkpoints.

Type II: pick up at checkpoints, and drop off not at checkpoints.

Type III: pick up not at checkpoints, and drop off at checkpoints.

3.2. Competing Transit Policies. To compare the performance of the two flexible transit systems, both route deviation policy and point policy are implemented with the same fleet size $M$ and passenger distribution. Bus cruising speed control strategy [23] is assumed to be implemented to eliminate the variation of demand locations. 


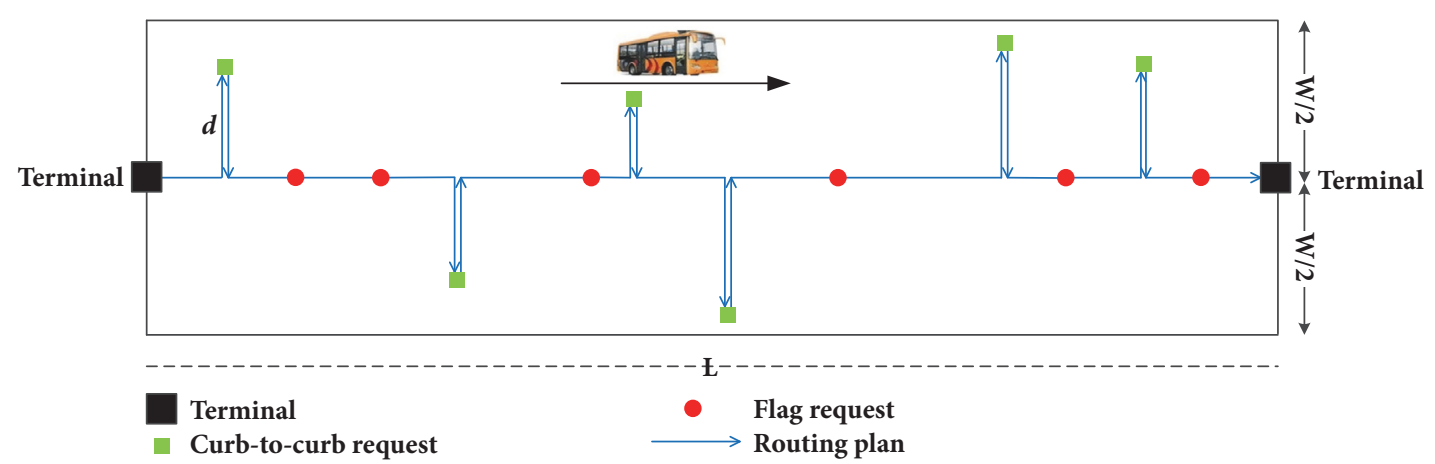

FIGURE 1: Route deviation operating policy.

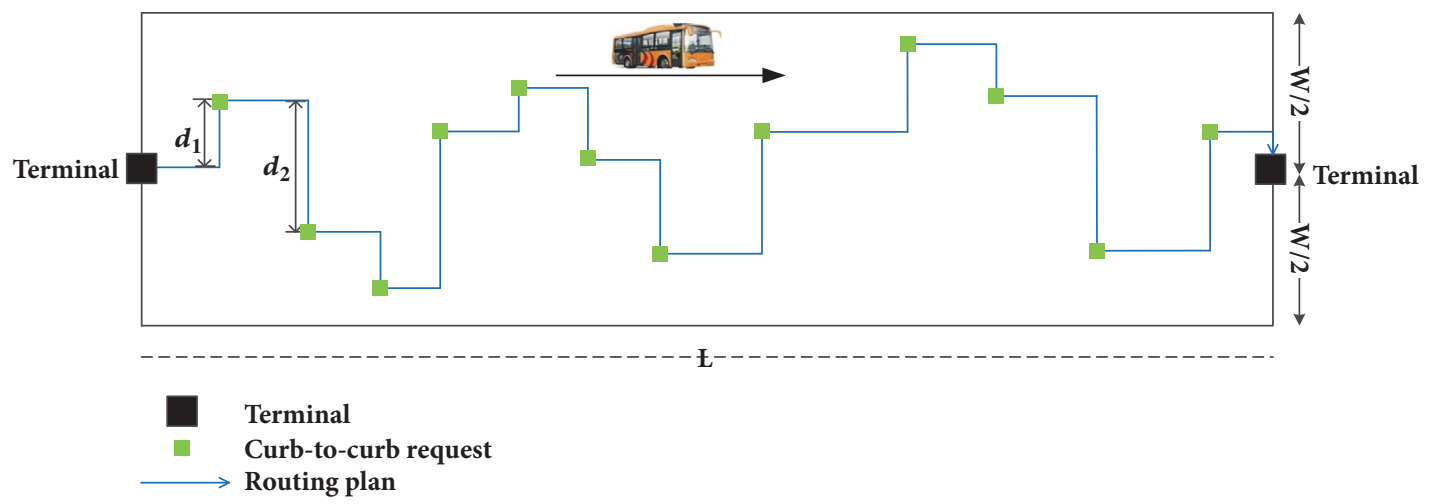

FIgURE 2: Point deviation operating policy.

3.2.1. Route Deviation Policy. As shown in Figure 1, the service vehicle operates a regular schedule along the base route and deviates to serve the curb-to-curb requests on either side of the base route with a maximum allowable deviation width of $W / 2$. Types II and III passengers can request the transit in two ways. The first kind of request is called a flag request in which passengers have a vertical walk between the base route and their house and the service vehicles stop to board or discharge passengers at any location requested along the base route. Another kind of request is a curb-to-curb request which requires passengers to make advance notice to schedule their noncheckpoint stops. After serving the curbto-curb passengers, the vehicle must return to the base route at the exact point where it left to accommodate the deviation.

The slack time for route deviation service is allotted based on the expected demand levels of the flag requests and curbto-curb requests. A no-rejection policy is applied for flag requests as the variation of travel demand has limited impact on the service cycle. The deviation service operates on a first come, first available basis. When the slack time is unable to accommodate the actual demand, some of the curb-to-curb customers who reserve later must be rejected due to the slack time limitation.

3.2.2. Point Deviation Policy. As another kind of flexible transit operating policy, service vehicles in point deviation system are not constrained to follow any predefined base route. Except for the time constraints of the checkpoints, the remainder of the service is demand-responsive and vehicles serve curb-to-curb requests within the service area (see Figure 2).

Given that the path between the scheduled stops might change in each ride, flag requests are not possible. Instead, all types II and III passengers are required to make reservations to schedule their noncheckpoint stops through smartphones or the Internet. Similar to route deviation system, there is also a predefined slack time based on the expected demand level. Accepted curb-to-curb requests have no walking in their trip. If the actual demand exceeds the expected demand level, rejections may occur for curb-to-curb passengers.

\section{Modeling}

In our study, passengers are assumed to be transit-dependent. Vehicle follows a rectilinear metric, which has been proven to be a good approximation of reality [24]. The services are provided by vehicles with the following characteristics: the cruising speed considering stops due to traffic and pedestrian interference, $V_{b}$; the dwelling time of a flag stop or a request stop, $T_{d}^{r}$; and dwelling time of a checkpoint, $T_{d}^{f}$. The walking speed of passenger is $V_{w k} . \theta$ and $\theta_{c}$ represent the expected demand rate (passenger/h) and actual demand rate (passenger/h) in the service area, respectively.

In practice, the actual travel demand frequently deviates from the expected demand level in low-demand service 


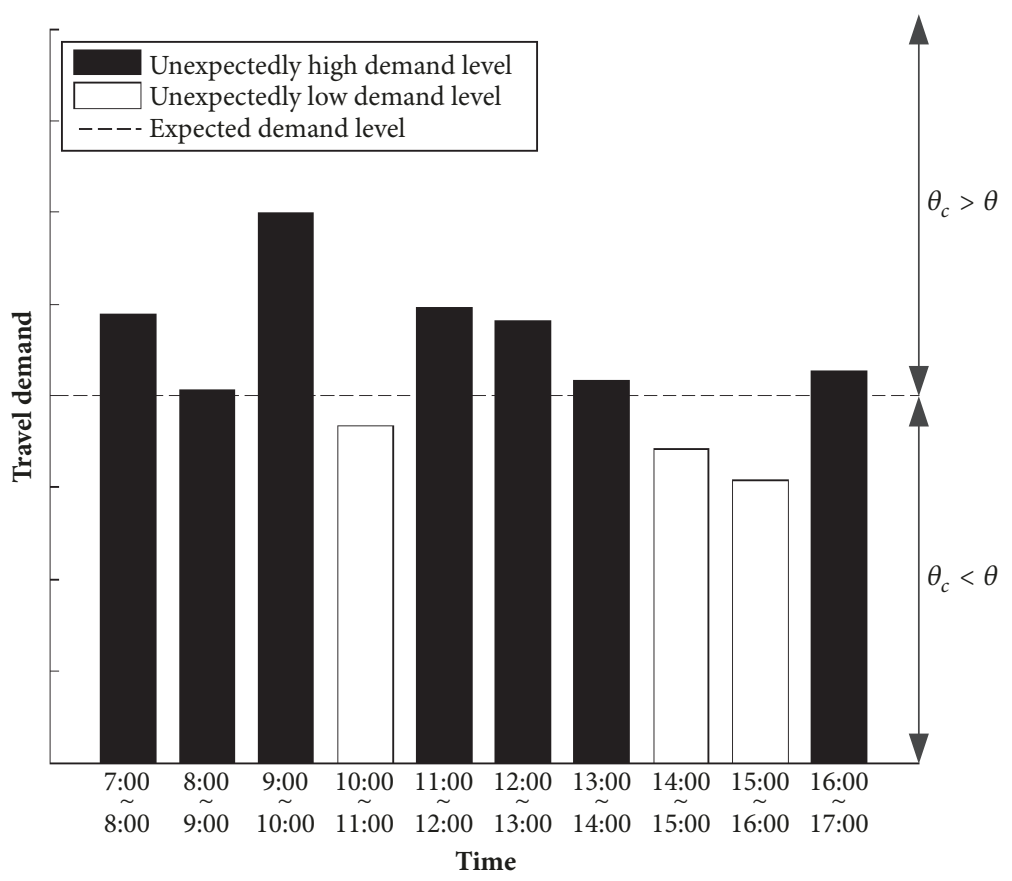

FIGURE 3: Uncertainties in travel demand.

areas due to uncertainties (see Figure 3). To investigate the performance of the two flexible transit systems, we measure the system performance at three demand levels, namely, the expected demand level, an unexpectedly high demand level, and an unexpectedly low-demand level.

4.1. Measure of Performance. There is no designed idle time between trips, and we keep the same fleet under different transit policies. If we disregard the difference in complexity of the reservation and scheduling systems, which only account for a small fraction of long-term transit operation, the two transit policies are considered have the same operating cost. Thus, in our analysis, the performance measure of the transit system is defined as the user cost function $F$, which reflects the service quality and greatly impacts the passengers' attitude towards the transit services. Similar to the previous study by Li and Quadrifoglio [25], the user cost function is constructed as the weighted sum of three indicators, the expected walking time $K$, the expected waiting time $A$, and the expected riding time $R$, with weights of $\omega_{K}, \omega_{A}$, and $\omega_{R}$, respectively. A lower value of $F$ clearly indicates a better transit service policy.

$$
F=\omega_{K} K+\omega_{A} A+\omega_{R} R
$$

4.2. Analytical Model of Route Deviation Service. For route deviation service, we assume the proportions of the curb-tocurb type II and type III passengers are $\alpha$ and $\beta$, respectively. Correspondingly, the proportions of the flag request type II and type III passengers are $1-\alpha$ and $1-\beta$.

4.2.1. At the Expected Demand Level. At the expect demand level, all curb-to-curb requests can be accepted. The expected vertical distance between the base route and the request stops (see Figure 1) is

$$
d=\frac{W}{4}
$$

Assuming a vehicle serves $k$ passenger in a single-trip time $T_{\mathrm{r}}$, the travel distance $D$ of a vehicle in one trip is

$$
D=L+\left(k \alpha \gamma_{2}+k \beta \gamma_{3}\right) \times 2 d=L+\frac{W\left(k \alpha \gamma_{2}+k \beta \gamma_{3}\right)}{2}
$$

In route deviation service, another two relationships can be obtained:

$$
\begin{aligned}
T_{\mathrm{r}} & =\frac{D}{V_{b}}+k\left(\gamma_{2}+\gamma_{3}\right) T_{d}^{r}+T_{d}^{f} \\
T_{\mathrm{r}} \times \theta & =k \times M
\end{aligned}
$$

Combining (3)-(5), we can derive the single-trip time $T_{\mathrm{r}}$ as follows:

$$
T_{\mathrm{r}}=\frac{2 M L+2 V_{b} M T_{d}^{f}}{2 M V_{b}-W \theta\left(\alpha \gamma_{2}+\beta \gamma_{3}\right)-2 \theta V_{b} T_{d}^{r}\left(\gamma_{2}+\gamma_{3}\right)}
$$

In the system, type I passengers do not walk as part of their trips $\left(K_{\mathrm{I}}=0\right)$. Curb-to-curb type II and type III passengers also have no waking time $K_{\mathrm{II}}^{\mathrm{c}}=K_{\mathrm{III}}^{\mathrm{c}}=0$. Flag request passengers walk vertically between their houses and base route, and their expected walking time is

$$
K_{\mathrm{II}}^{\mathrm{f}}=K_{\mathrm{III}}^{\mathrm{f}}=\frac{W}{4 V_{w k}}
$$


Thus, the expected walking time per passenger is

$$
\begin{aligned}
K= & \gamma_{1} K_{\mathrm{I}}+\gamma_{2}\left(\alpha K_{\mathrm{II}}^{\mathrm{c}}+(1-\alpha) K_{\mathrm{II}}^{\mathrm{f}}\right) \\
& +\gamma_{3}\left(\beta K_{\mathrm{III}}^{\mathrm{c}}+(1-\beta) K_{\mathrm{III}}^{\mathrm{f}}\right) \\
= & \left(\gamma_{2}+\gamma_{3}-\alpha \gamma_{2}-\beta \gamma_{3}\right) \frac{W}{4 V_{w k}}
\end{aligned}
$$

The headway of route deviation system is $2 T_{\mathrm{r}} / M$, and the expected waiting time of the type I, type II, and flag request type III passengers is

$$
A_{\mathrm{I}}=A_{\mathrm{II}}=A_{\mathrm{III}}^{\mathrm{f}}=\frac{T_{\mathrm{r}}}{M}
$$

Curb-to-curb type III passengers can receive a scheduled pick-up time from the system when they make advanced notice. They are more likely to spend their time at their house or comfortable locations instead of waiting outside before the scheduled pick-up time. Therefore, their waiting time is defined as the interval between the scheduled pickup time and the actual pick-up time. The waiting time for a curb-to-curb type III passenger $i$ consists of two parts. The first part is the sum of service time of the flag requests before the vehicle picks up $i$. The second part is the follow-up curb-to-curb customers who make reservations later than $i$ causing additional stops before the vehicle picks up $i$. Based on mathematical probability theory, the expected value of the waiting time can be expressed as

$$
\begin{aligned}
A_{\mathrm{III}}^{c}= & \frac{\sum_{i=1}^{k\left(\alpha \gamma_{2}+\beta \gamma_{3}\right)-1} i}{k\left(\alpha \gamma_{2}+\beta \gamma_{3}\right)}\left(\frac{W}{2 V_{b}}+T_{d}^{r}\right) \frac{1}{2} \\
& +\frac{k\left((1-\alpha) \gamma_{2}+(1-\beta) \gamma_{3}\right)}{2} T_{d}^{r} \\
= & \frac{W k\left(\alpha \gamma_{2}+\beta \gamma_{3}\right)}{8 V_{b}}+\frac{T_{d}^{r} k\left(\alpha \gamma_{2}+\beta \gamma_{3}\right)}{4}-\frac{W}{8 V_{b}} \\
& -\frac{T_{d}^{r}}{4}+\frac{T_{d}^{r} k\left((1-\alpha) \gamma_{2}+(1-\beta) \gamma_{3}\right)}{2}
\end{aligned}
$$

Thus, the expected waiting time per passenger is

$$
\begin{aligned}
A= & \gamma_{1} A_{\mathrm{I}}+\gamma_{2} A_{\mathrm{II}}+\gamma_{3}\left(\beta A_{\mathrm{III}}^{c}+(1-\beta) A_{\mathrm{III}}^{\mathrm{f}}\right) \\
= & \frac{\left(2 L+2 V_{b} T_{d}^{f}\right)\left(\gamma_{1}+\gamma_{2}+\gamma_{3}(1-\beta)\right)}{2 M V_{b}-W \theta\left(\alpha \gamma_{2}+\beta \gamma_{3}\right)-2 \theta V_{b} T_{d}^{r}\left(\gamma_{2}+\gamma_{3}\right)} \\
& +\frac{\gamma_{3} \beta W k\left(\alpha \gamma_{2}+\beta \gamma_{3}\right)-\gamma_{3} \beta W}{8 V_{b}} \\
& +\frac{\gamma_{3} \beta T_{d}^{r} k\left(\alpha \gamma_{2}+\beta \gamma_{3}\right)-\gamma_{3} \beta T_{d}^{r}}{4} \\
& +\frac{\gamma_{3} \beta T_{d}^{r} k\left((1-\alpha) \gamma_{2}+(1-\beta) \gamma_{3}\right)}{2}
\end{aligned}
$$

Type I passengers travel from one terminal to another, so their expected riding time is

$$
R_{\mathrm{I}}=T_{\mathrm{r}}
$$

Types II and III passengers can be dropped off or picked up uniformly anytime in the trip between two terminals, and their expected riding time can be expressed as

$$
R_{\mathrm{II}}=R_{\mathrm{III}}=\frac{T_{\mathrm{r}}}{2}
$$

Then, the expected riding time per passenger is

$$
\begin{aligned}
R & =\gamma_{1} R_{\mathrm{I}}+\gamma_{2} R_{\mathrm{II}}+\gamma_{3} R_{\mathrm{III}} \\
& =\frac{\left(M L+V_{b} M T_{d}^{f}\right)\left(1+\gamma_{1}\right)}{2 M V_{b}-W \theta\left(\alpha \gamma_{2}+\beta \gamma_{3}\right)-2 \theta V_{b} T_{d}^{r}\left(\gamma_{2}+\gamma_{3}\right)}
\end{aligned}
$$

4.2.2. At Unexpectedly Low-Demand Level. When $\theta_{c}<\theta$, there will be idle time at the downstream checkpoint. For this condition, the expected walking time is equal to the result from (8). The expected waiting of the type I, type II, and flag request type III passengers can be derived by (9).

For each ride, the actual served passengers can be expressed as

$$
\begin{aligned}
k_{c} & =\frac{\theta_{c} \times T_{r}}{M} \\
& =\frac{2 \theta_{c} L+2 V_{b} \theta_{c} T_{d}^{f}}{2 M V_{b}-W \theta\left(\alpha \gamma_{2}+\beta \gamma_{3}\right)-2 \theta V_{b} T_{d}^{r}\left(\gamma_{2}+\gamma_{3}\right)}
\end{aligned}
$$

The expected waiting time of curb-to-curb type III passengers can be obtained by replacing $k$ with $k_{c}$ in (10). Then, we can obtain the expected waiting time at unexpectedly lowdemand levels by $A=\gamma_{1} A_{\mathrm{I}}+\gamma_{2} A_{\mathrm{II}}+\gamma_{3}\left(\beta A_{\mathrm{III}}^{c}+(1-\beta) A_{\mathrm{III}}^{f}\right)$.

At unexpectedly low-demand levels, there will be idle time at the terminal checkpoints. The expected riding times of different types of passengers can be obtained as follows:

$$
\begin{aligned}
R_{\mathrm{I}}= & \frac{L+W\left(k_{\mathrm{c}} \alpha \gamma_{2}+k_{\mathrm{c}} \beta \gamma_{3}\right) / 2}{V_{b}}+k_{\mathrm{c}}\left(\gamma_{2}+\gamma_{3}\right) T_{d}^{r} \\
& +T_{d}^{f} \\
R_{\mathrm{II}}= & R_{\mathrm{III}} \\
= & \frac{L+W\left(k_{\mathrm{c}} \alpha \gamma_{2}+k_{\mathrm{c}} \beta \gamma_{3}\right) / 2}{2 V_{b}}+\frac{k_{\mathrm{c}}\left(\gamma_{2}+\gamma_{3}\right)}{2} T_{d}^{r} \\
& +\frac{T_{d}^{f}}{2}
\end{aligned}
$$

Thus, the expected riding time per passenger at unexpectedly low-demand levels can be expressed as

$$
\begin{aligned}
R= & \gamma_{1} R_{\mathrm{I}}+\gamma_{2} R_{\mathrm{II}}+\gamma_{3} R_{\mathrm{III}}=\left(1+\gamma_{1}\right)\left[\frac{L+V_{b} T_{d}^{f}}{2 V_{b}}\right. \\
& \left.+\left(\frac{W\left(\alpha \gamma_{2}+\beta \gamma_{3}\right)}{4 V_{b}}+\frac{T_{d}^{r}\left(\gamma_{2}+\gamma_{3}\right)}{2}\right) k_{c}\right]
\end{aligned}
$$


4.2.3. At Unexpectedly High Demand Level. When the actual demand exceeds the service capacity of deviation services, some of the curb-to-curb requests have to be rejected due to slack time limitation. These rejected customers may need to wait more than one operating cycle before pick-up. However, waiting for the next available vehicle makes it difficult for the rejected passengers to follow their schedules. Thus, we assume that rejected curb-to-curb passengers become flag request passengers for desired transit service.

When $\theta_{c}>\theta$, the first $\theta$ passengers can be accepted, and the values of the passenger cost indicators are the same as those at the expected demand level. For the last $\theta_{\mathrm{c}}-\theta$ passengers, type I and flag request types II and III passengers can be accepted and the actual number of rejected request is $\left(\theta_{c}-\theta\right)\left(\alpha \gamma_{2}+\beta \gamma_{3}\right)$. For these $\theta_{c}-\theta$ passengers, $K_{\mathrm{I}}^{\theta_{c}-\theta}=0$, and the expected walking time of types II and III passengers is

$$
K_{\mathrm{II}}^{\theta_{c}-\theta}=K_{\mathrm{III}}^{\theta_{c}-\theta}=\frac{W}{4 V_{w k}}
$$

We define $\delta=K$ in (8), and the expected walking time of all passengers can be calculated as

$$
K=\frac{\theta}{\theta_{c}} \delta+\frac{\left(\theta_{c}-\theta\right)\left(\gamma_{2}+\gamma_{3}\right) W}{4 V_{w k} \theta_{c}}
$$

Because of the no-rejection policy, the last $\left(\theta_{c}-\theta\right)\left(\gamma_{2}+\gamma_{3}\right)$ flag request passengers prolong the single-trip time. Thus, the actual single-trip time $T_{r}^{a}$ is

$$
T_{r}^{a}=T_{r}+\left(k_{c}-k\right)\left(\gamma_{2}+\gamma_{3}\right) T_{d}^{r}
$$

At unexpectedly high demand levels, the expected waiting of the type I, type II, and flag request type III passengers can be calculated by replacing $T_{r}^{a}$ with $T_{r}$ in (9). The expected waiting time of curb-to-curb type III passengers can be expressed as

$$
\begin{aligned}
A_{\mathrm{III}}^{c} & =\frac{\sum_{i=1}^{k\left(\alpha \gamma_{2}+\beta \gamma_{3}\right)-1} i}{k\left(\alpha \gamma_{2}+\beta \gamma_{3}\right)}\left(\frac{W}{2 V_{b}}+T_{d}^{r}\right) \frac{1}{2} \\
+ & \frac{k\left((1-\alpha) \gamma_{2}+(1-\beta) \gamma_{3}\right)+\left(k_{c}-k\right)\left(\gamma_{2}+\gamma_{3}\right)}{2} \\
\cdot & T_{d}^{r}=\frac{W k\left(\alpha \gamma_{2}+\beta \gamma_{3}\right)}{8 V_{b}}+\frac{T_{d}^{r} k\left(\alpha \gamma_{2}+\beta \gamma_{3}\right)}{4}-\frac{W}{8 V_{b}} \\
- & \frac{T_{d}^{r}}{4}+\frac{T_{d}^{r}\left(k_{c}\left(\gamma_{2}+\gamma_{3}\right)-k \alpha \gamma_{2}-k \beta \gamma_{3}\right)}{2}
\end{aligned}
$$

Thus, the expected waiting time of all passengers is

$$
A=\gamma_{1} A_{\mathrm{I}}+\gamma_{2} A_{\mathrm{II}}+\gamma_{3}\left(\frac{k \beta}{k_{c}} A_{\mathrm{III}}^{\mathrm{c}}+\frac{k_{c}-k \beta}{k_{c}} A_{\mathrm{III}}^{\mathrm{f}}\right)
$$

Similarly, the expected riding time of type I passengers is $T_{r}^{a}$ and the expected riding time of types II and III passengers is $T_{r}^{a} / 2$; thus, the expected riding time per passenger is

$$
\begin{aligned}
R & =\gamma_{1} R_{\mathrm{I}}+\gamma_{2} R_{\mathrm{II}}+\gamma_{3} R_{\mathrm{III}} \\
& =\left(1+\gamma_{1}\right) \frac{T_{r}+\left(k_{c}-k\right)\left(\gamma_{2}+\gamma_{3}\right) T_{d}^{r}}{2}
\end{aligned}
$$

4.3. Analytical Model of Point Deviation Service. For point deviation service, a "no-backtracking constraint policy" is applied, which forbids backward movements of the service vehicles; therefore, passengers are served in order of horizontal coordinates [26]. This routing policy may not be optimal but it is easy to implement, especially in complex road networks.

4.3.1. At the Expected Demand Level. At the expected demand level, we assume an ideal case in which no rejection occurs $(K=0)$ and the downstream terminal checkpoint features no idle time. As shown in Figure 2, let $d_{1}$ indicate the vertical distance between the terminal checkpoints and the fist/last on-request stop. We have that $\mathrm{E}\left(d_{1}\right)=W / 4$. Let $d_{2}$ indicate the vertical distance between any pair of on-request stops; we obtain that $\mathrm{E}\left(d_{2}\right)=W / 3$.

Similar to the previous analytical method for point deviation service, the following relationships can be obtained:

$$
\begin{aligned}
D & =L+2 \times d_{1}+\left[k\left(\gamma_{2}+\gamma_{3}\right)-1\right] \times d_{2} \\
& =L+\frac{W}{6}+\frac{k W\left(\gamma_{2}+\gamma_{3}\right)}{3} \\
T_{\mathrm{r}} & =\frac{D}{V_{b}}+k\left(\gamma_{2}+\gamma_{3}\right) T_{d}^{r}+T_{d}^{f} \\
T_{\mathrm{r}} \times \theta & =k \times M
\end{aligned}
$$

Combining (25)-(27), the single-trip time $T_{\mathrm{r}}$ can be derived as follows:

$$
T_{\mathrm{r}}=\frac{6 M L+M W+6 M V_{b} T_{d}^{f}}{6 M V_{b}-2 W \theta\left(\gamma_{2}+\gamma_{3}\right)-6 \theta V_{b} T_{d}^{r}\left(\gamma_{2}+\gamma_{3}\right)}
$$

The expected waiting time of different types of passenger can be expressed as

$$
\begin{aligned}
A_{\text {I }} & =A_{\text {II }}=\frac{T_{\mathrm{r}}}{M} \\
A_{\text {III }} & =\frac{\sum_{i=1}^{k\left(\gamma_{2}+\gamma_{3}\right)-1} i}{k\left(\gamma_{2}+\gamma_{3}\right)}\left(\frac{W}{3 V_{b}}+T_{d}^{r}\right) \frac{1}{2} \\
& =\frac{W k\left(\gamma_{2}+\gamma_{3}\right)}{12 V_{b}}+\frac{T_{d}^{r} k\left(\gamma_{2}+\gamma_{3}\right)}{4}-\frac{W}{12 V_{b}}-\frac{T_{d}^{r}}{4}
\end{aligned}
$$

Thus, the expected value of waiting time per passenger is

$$
\begin{aligned}
A= & \gamma_{1} A_{\mathrm{I}}+\gamma_{2} A_{\mathrm{II}}+\gamma_{3} A_{\mathrm{III}} \\
= & \frac{\left(6 L+W+6 V_{b} T_{d}^{f}\right)\left(\gamma_{1}+\gamma_{2}\right)}{6 M V_{b}-2 W \theta\left(\gamma_{2}+\gamma_{3}\right)-6 \theta V_{b} T_{d}^{r}\left(\gamma_{2}+\gamma_{3}\right)} \\
& +\frac{\gamma_{3} W k\left(\gamma_{2}+\gamma_{3}\right)-\gamma_{3} W}{12 V_{b}} \\
& +\frac{\gamma_{3} T_{d}^{r} k\left(\gamma_{2}+\gamma_{3}\right)-\gamma_{3} T_{d}^{r}}{4}
\end{aligned}
$$

Just as in the route deviation service, the expected riding time of type I passengers is $T_{\mathrm{r}}$ and the expected riding time of 
types II and III passengers is $T_{\mathrm{r}} / 2$. Thus, the expected riding time per passengers can be calculated as

$$
\begin{aligned}
R & =\gamma_{1} R_{\mathrm{I}}+\gamma_{2} R_{\mathrm{II}}+\gamma_{3} R_{\mathrm{III}} \\
& =\frac{\left(6 M L+M W+6 M V_{b} T_{d}^{f}\right)\left(1+\gamma_{1}\right)}{12 M V_{b}-4 W \theta\left(\gamma_{2}+\gamma_{3}\right)-12 \theta V_{b} T_{d}^{r}\left(\gamma_{2}+\gamma_{3}\right)}
\end{aligned}
$$

4.3.2. At Unexpectedly Low-Demand Level. If $\theta_{c}<\theta$, no passengers are rejected by the system $(K=0)$. In this case, the expected waiting time of types I and II passengers can be calculated by (29). The expected waiting of the types III passengers can be calculated by replacing $k$ with $k_{\mathrm{c}}$ $\left(k_{\mathrm{c}}=\theta_{c} T_{\mathrm{r}} / M\right)$ in (30). Then, we can determine the expected waiting time per passenger as $A=\gamma_{1} A_{\mathrm{I}}+\gamma_{2} A_{\mathrm{II}}+\gamma_{3} A_{\mathrm{III}}$.

As the actual demand is lower than expected, the vehicle will arrive earlier than the schedule arrival time. The expected riding times of different types of passengers are

$$
\begin{aligned}
R_{\mathrm{I}}= & \frac{L}{V_{\mathrm{b}}}+\frac{W}{6 V_{\mathrm{b}}}+\frac{k_{\mathrm{c}} W\left(\gamma_{2}+\gamma_{3}\right)}{3 V_{\mathrm{b}}}+k_{\mathrm{c}}\left(\gamma_{2}+\gamma_{3}\right) T_{d}^{r} \\
& +T_{d}^{f} \\
R_{\mathrm{II}}= & R_{\mathrm{III}} \\
= & \frac{L}{2 V_{\mathrm{b}}}+\frac{W}{12 V_{\mathrm{b}}}+\frac{k_{\mathrm{c}} W\left(\gamma_{2}+\gamma_{3}\right)}{6 V_{\mathrm{b}}}+\frac{k_{\mathrm{c}}\left(\gamma_{2}+\gamma_{3}\right) T_{d}^{r}}{2} \\
& +\frac{T_{d}^{f}}{2}
\end{aligned}
$$

Thus, the expected riding time per passenger at unexpectedly low-demand levels can be expressed as

$$
\begin{aligned}
R= & \gamma_{1} R_{\mathrm{I}}+\gamma_{2} R_{\mathrm{II}}+\gamma_{3} R_{\mathrm{III}}=\left(1+\gamma_{1}\right)\left[\frac{L}{2 V_{\mathrm{b}}}+\frac{W}{12 V_{\mathrm{b}}}\right. \\
& \left.+\frac{k_{\mathrm{c}} W\left(\gamma_{2}+\gamma_{3}\right)}{6 V_{\mathrm{b}}}+\frac{k_{\mathrm{c}}\left(\gamma_{2}+\gamma_{3}\right) T_{d}^{r}}{2}+\frac{T_{d}^{f}}{2}\right]
\end{aligned}
$$

4.3.3. At Unexpectedly High Demand Level. Unlike route deviation service, in point deviation service, the rejected passengers cannot issue a flag request since there is no base route for the service. Therefore, we assume that rejected customers utilize the nearest checkpoint for transit. Generally, curb-to-curb passengers are required to request service at least two hours in advance. Thus, the rejected passengers are considered to have sufficient time to reach the checkpoint before pick up.

For the last $\theta_{c}-\theta$ passengers, type I passengers can be accepted and the rejected curb-to-curb request is $\left(\theta_{c}-\theta\right)\left(\gamma_{2}+\right.$ $\left.\gamma_{3}\right)$. The expected walking time of types II and III passengers is

$$
K_{\mathrm{II}}^{\theta_{c}-\theta}=K_{\mathrm{III}}^{\theta_{c}-\theta}=\frac{L}{4 V_{w k}}+\frac{W}{4 V_{w k}}
$$

TABLE 1: Parameter values.

\begin{tabular}{lc}
\hline Parameter & Value \\
\hline$L$ & 3 miles \\
$W$ & 1 miles \\
$M$ & 1 \\
$V_{b}$ & $25 \mathrm{miles} / \mathrm{h}$ \\
$V_{w k}$ & $3 \mathrm{miles} / \mathrm{h}$ \\
$T_{d}^{r}$ & $12 \mathrm{sec}$ \\
$T_{d}^{f}$ & $15 \mathrm{sec}$ \\
$\gamma_{1} / \gamma_{2} / \gamma_{3}$ & $0.2 / 0.4 / 0.4$ \\
\hline
\end{tabular}

Thus, the expected walking time per passenger can be expressed as

$$
K=\frac{\left(\theta_{c}-\theta\right)\left(\gamma_{2}+\gamma_{3}\right)(L+W)}{4 V_{w k} \theta_{c}}
$$

For the last $\theta_{c}-\theta$ passengers, the expected waiting time of type I passengers is $A_{\mathrm{I}}^{\theta_{c}-\theta}=T_{r} / M$. As the rejected type III passengers are informed about the transit schedule, $A_{\mathrm{III}}^{\theta_{c}-\theta}=$ 0 . Rejected type II passengers either walk directly to their destinations or obtain a ride to the downstream checkpoint; thus the expected waiting time can be derived as

$$
A_{\mathrm{II}}^{\theta_{c}-\theta}=\frac{1}{2} \times 0+\frac{1}{2} \times \frac{T_{r}}{M}=\frac{T_{r}}{2 M}
$$

We define $\lambda=A$ in (31), and the expected waiting time of all passengers can be derived as

$$
A=\frac{\theta}{\theta_{c}} \lambda+\frac{\gamma_{1}\left(\theta_{c}-\theta\right) T_{r}}{M \theta_{c}}+\frac{\gamma_{2}\left(\theta_{c}-\theta\right) T_{r}}{2 M \theta_{c}}
$$

Similarly, in the last $\theta_{c}-\theta$ passengers, the expected riding time of type I passengers is $R_{\mathrm{I}}^{\theta_{c}-\theta}=T_{r}$. For types II and III passengers, the expected riding time can be expressed as

$$
R_{\mathrm{II}}^{\theta_{c}-\theta}=R_{\mathrm{III}}^{\theta_{c}-\theta}=\frac{1}{2} \times 0+\frac{1}{2} \times T_{r}=\frac{T_{r}}{2}
$$

We can observe that the expected riding time of the $\theta_{c}-\theta$ passengers remains unchanged; therefore the expected riding time of all passengers can be calculated by (32).

\section{Result Analysis}

5.1. Parameter Values. In this section, a case study is conducted based the Route 289 service in the suburban area of Zhengzhou City in China which currently operates under a pure flag stop policy [18]. In this paper, we use the system parameters and operating data to test system performance under both route deviation policy and point deviation policy. The parameter values for the analysis are presented in Table 1. The weights values $\left(\omega_{K}, \omega_{A}\right.$, and $\left.\omega_{R}\right)$ in the user cost function may be varied according to a wide range of socioeconomic and environment factors. Similar to the previous research, we assume that $\omega_{A}=1$ and $\omega_{R}=1$ [16] and the default weight of the walking time is set to $\omega_{K}=2$ [27]. Cases with different weights will be discussed in sensitivity analysis. 
TABLE 2: Passenger cost indicators in route deviation service $(\alpha=0.1$ and $\beta=0.1)$.

\begin{tabular}{lccccccc}
\hline Indicator (min) & & \multicolumn{5}{c}{ Demand (passengers/h) } \\
& 26 & 30 & 34 & 38 & 42 & 46 \\
\hline$T_{\mathrm{r}}$ & 8.38 & 8.54 & 8.71 & 8.89 & 9.08 & 9.27 \\
$K$ & 3.60 & 3.60 & 3.60 & 3.60 & 3.60 & 3.60 & 3.60 \\
$A$ & 8.04 & 8.20 & 8.37 & 8.54 & 8.72 & 8.91 & 9.11 \\
$R$ & 5.03 & 5.13 & 5.23 & 5.33 & 5.45 & 5.56 \\
$F$ & 20.27 & 20.53 & 20.80 & 21.08 & 21.37 & 21.67 \\
\hline
\end{tabular}

TABle 3: Passenger cost indicators in point deviation service.

\begin{tabular}{|c|c|c|c|c|c|c|c|}
\hline \multirow{2}{*}{ Indicator (min) } & \multicolumn{7}{|c|}{ Demand (passengers/h) } \\
\hline & 26 & 30 & 34 & 38 & 42 & 46 & 50 \\
\hline$T_{\mathrm{r}}$ & 12.02 & 13.08 & 14.36 & 15.91 & 17.84 & 20.30 & 23.55 \\
\hline$K$ & 0.00 & 0.00 & 0.00 & 0.00 & 0.00 & 0.00 & 0.00 \\
\hline$A$ & 7.53 & 8.27 & 9.17 & 10.25 & 11.60 & 13.33 & 15.60 \\
\hline$R$ & 7.21 & 7.85 & 8.62 & 9.55 & 10.70 & 12.18 & 14.13 \\
\hline$F$ & 14.73 & 16.12 & 17.78 & 19.80 & 22.31 & 25.51 & 29.73 \\
\hline
\end{tabular}

\subsection{Comparison of System Performance at the Expected} Demand Level. At the expected demand levels, the passenger cost indicators from the analytical models of both route deviation policy and point deviation policy are presented in Tables 2 and 3, respectively. The results illustrate that point deviation service outperforms route deviation service when the travel demand is low because there is no walking $(K=0)$ in the system. However, point deviation service is much more sensitive to the demand levels. The single-trip time $T_{\mathrm{r}}$ sharply increases with increasing demand under point deviation policy, which likely the result of long detours required by the curb-to-curb services. In contrast, the demand has limited influence on route deviation service $(\alpha=0.1$ and $\beta=0.1)$. Consequently, the passenger cost function of point deviation service enlarged dramatically due to the rapidly increasing values of $A$ and $R$ and overtakes route deviation service with the critical demand $\theta=42$ passengers $/ \mathrm{h}$.

To test the influence of the curb-to-curb passengers' proportions, the user cost functions with different $\alpha$ and $\beta$ are presented in Figure 4 . The results indicate that, for any proportions of $\alpha$ and $\beta$, point deviation service has a clear advantage in relatively low demand (e.g., $\theta<38$ passengers $/ \mathrm{h}$ ). With an increasing proportion of curb-tocurb passengers $(\alpha$ and $\beta$ ), the user function $F$ becomes more sensitive to the travel demand and the critical demand between the two policies becomes larger. When $\alpha=\beta=0.7$, point deviation service outperforms route deviation service under all demand levels. This is because, under route deviation policy, the vehicle has to return to the base route after serving a curb-to-curb passenger which becomes inefficient with a large proportion of curb-to-curb passengers. Besides, route deviation service with a large proportion of curb-to curb type III passengers $(\alpha=0.1, \beta=0.7)$ has a lower cost function than its counterparts $(\alpha=0.4, \beta=0.4)$. Although the two cases have the same number of curb-to curb passengers $\left(\alpha \gamma_{2}+\beta \gamma_{3}\right)$, curb-to curb type III passengers have shorter waiting time than those waiting at the terminals

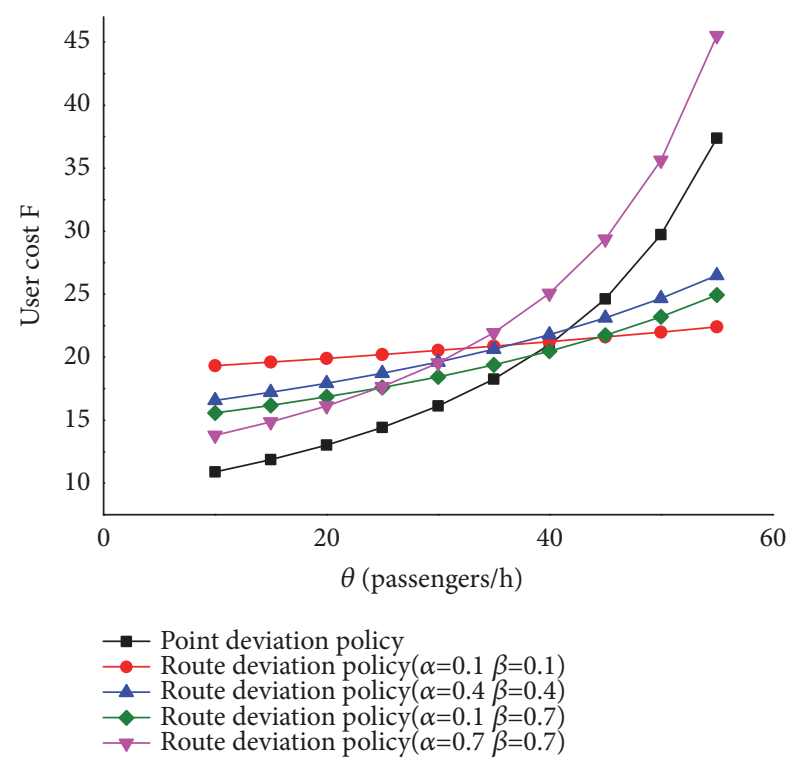

FIGURE 4: Comparison of system performance at the expected demand levels.

because of the advanced scheduled pick-up time notice. We can also observe that growth trends of the user cost for the two cases $(\alpha=0.1, \beta=0.7$, and $\alpha=\beta=0.4$ ) are almost the same which indicate that, for any other combinations of $\alpha$ and $\beta$, the growth trend is within the range of these three selected combinations. Hence, we use $\alpha=\beta=0.1, \alpha=\beta=0.4$, and $\alpha=\beta=0.7$ as typical cases in this study.

The above results confirm that, in the designed demand range, point deviation service is desirable at low-demand levels. The difference in system performance narrows between the two policies with the increasing demand. Route deviation service is more suitable for the relatively higher demand levels. With an increase in the proportion of curb-to-curb 


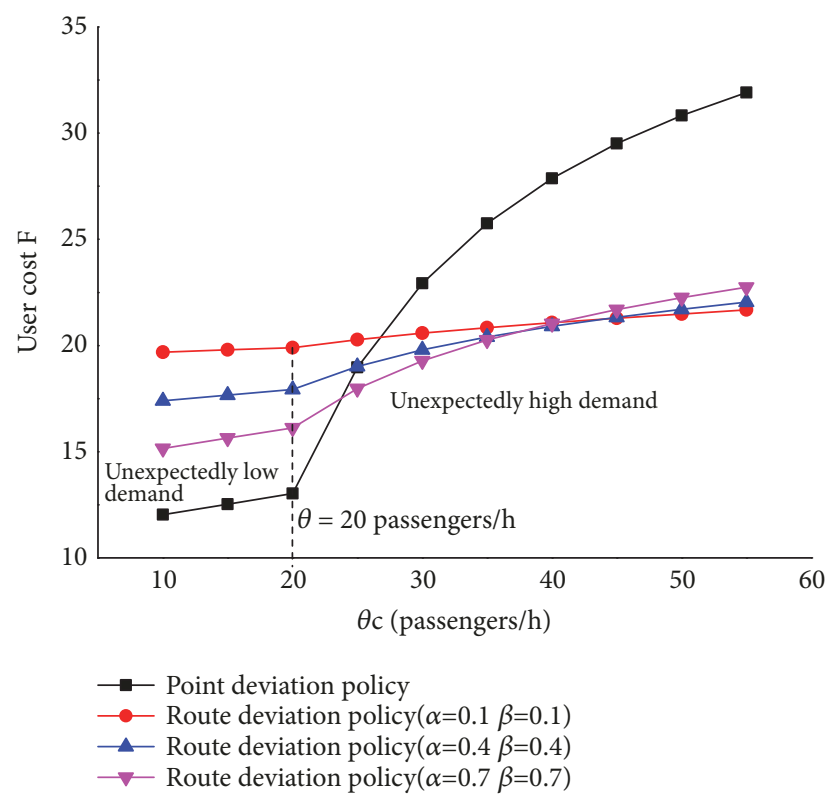

Figure 5: Comparison of system performance at unexpected demand levels.

passengers, route deviation service becomes more flexible but more sensitive to the demand. When this proportion is considerably large, point deviation service always performs better than route deviation service.

\subsection{Comparison of System Performance at Unexpected} Demand Levels. To test the reliability of the two transit services, the system performance at unexpected demand levels is presented in Figure 5 assuming an expected demand rate of $\theta=20$ passengers $/ \mathrm{h}$. The results illustrate that both services experience a slight decrease at unexpectedly lowdemand levels. The decreasing trend becomes more apparent with an increasing proportion of on-demand requests.

However, at unexpectedly high demand levels, the user cost of point deviation service surges with increasing actual demand $\theta_{c}$. The system performance of route deviation service is relatively more stable especially when $\alpha$ and $\beta$ are small. This result occurs because the passengers rejected in point deviation service have to walk to the nearest checkpoints while in route deviation service, rejected curbto curb passengers can simply walk directly to the base route and issue flag requests.

Based on these observations, we can conclude that route deviation service is more reliable than point deviation service and is more suitable to apply in the service areas with great demand variations.

5.4. Sensitivity Analysis. Sensitivity analysis is conducted under both expected and unexpected demand levels to examine how the performance of the two competing transit polices is affected by key input parameters. The results are presented in Figure 6. The weights of waiting time and walking time may vary in different external operation environments. To study the influence of these weights on the system performance, we consider a case where $\omega_{K}=3, \omega_{A}=3, \omega_{R}=1$.
This may occur in cases where walking and waiting are highly undesirable, such as under extreme weather or in unsafe neighborhood. As shown in Figure 6(a), the user costs increase under both policies, and the margin becomes more remarkable than in the base case. The critical demand between the two systems becomes larger, which suggests that point deviation service has a larger suitable demand range in a bad operation environment. At unexpectedly high demand levels, the difference in the user cost between the two policies becomes larger since walking is more unendurable in this case (see Figure 6(b)).

Another experiment with a long length ( $L=8$ miles) is conducted (see Figures 6(c) and 6(d)). In this case, the waiting time and riding time are enlarged because of the long travel distance. The critical demand is smaller than that in the base case which indicates that route deviation service has a large application demand range for long distance route. At unexpectedly high demand levels, the difference becomes more remarkable because the rejected curb-to-curb passengers in point deviation service have to spend more time walking to the nearest checkpoints.

In real-life cases, the permitted deviation ranges from 0.25 to 1.5 miles [4]. A larger width ( $W=1.5$ miles) is investigated, and the results are presented in Figures 6(e) and $6(f)$. In this case, route deviation service with a small proportion of curb-to-curb passenger has a slight increase in the user cost. In contrast, the user cost of point deviation service experiences a dramatic increase with increasing demand due to the longer travel distance needed to travel to serve curb-tocurb requests. The switching point of the two transits is at a lower demand level, which demonstrates that point deviation service is quite sensitive to the width of the service area and it is more favorable to operate in narrowed service area.

Furthermore, a two-vehicle case $(M=2)$ is investigated, and the results are displayed in Figures 6(g) and 6(h). The two vehicles run along the base route in opposite directions, and they leave the two terminals at the same time. The outcomes indicate that point deviation service is more sensitive to the fleet size and has a better performance under lowto-moderate demand levels. At unexpectedly high demand levels, route deviation service shows better reliability than that in the base case. This result illustrates that more available vehicles can improve the robustness of route deviation service.

\section{Conclusion}

This paper helps planners to select the most appropriate flexible transit services in low-demand areas. Route deviation service and point deviation service are chosen as typical flexible policies in this study. The former is the most widely used in practice, while the latter has been extensively investigated by researchers.

The two flexible systems differ in operation rules and degree of flexibility offered. Generally, route deviations systems are much more constrained than those allowed for point deviation systems. By modeling a real-life transit service, we compare the system performance of the two competing transit policies under both expected and unexpected demands. 


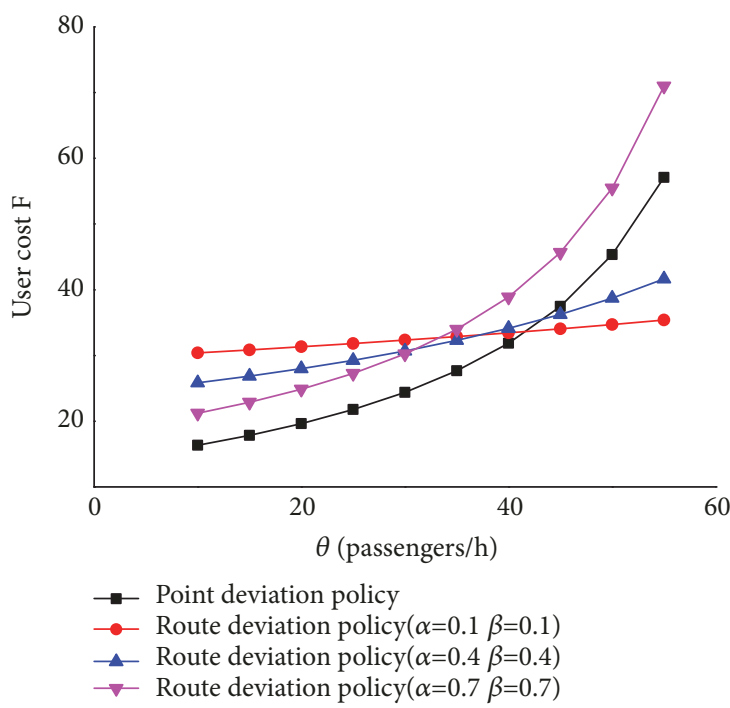

(a) At expected demand $\left(\omega_{K}=3, \omega_{A}=3, \omega_{R}=1\right)$

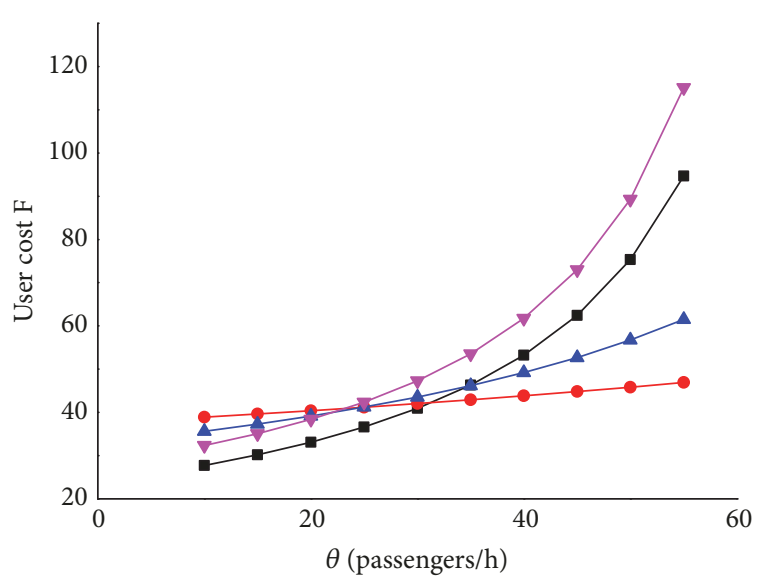

$\rightarrow$ Point deviation policy

$\rightarrow$ Route deviation policy $(\alpha=0.1 \quad \beta=0.1)$

$\_$Route deviation policy $(\alpha=0.4 \beta=0.4)$

$\neg-$ Route deviation policy $(\alpha=0.7 \beta=0.7)$

(c) At expected demand ( $L=8$ mile)

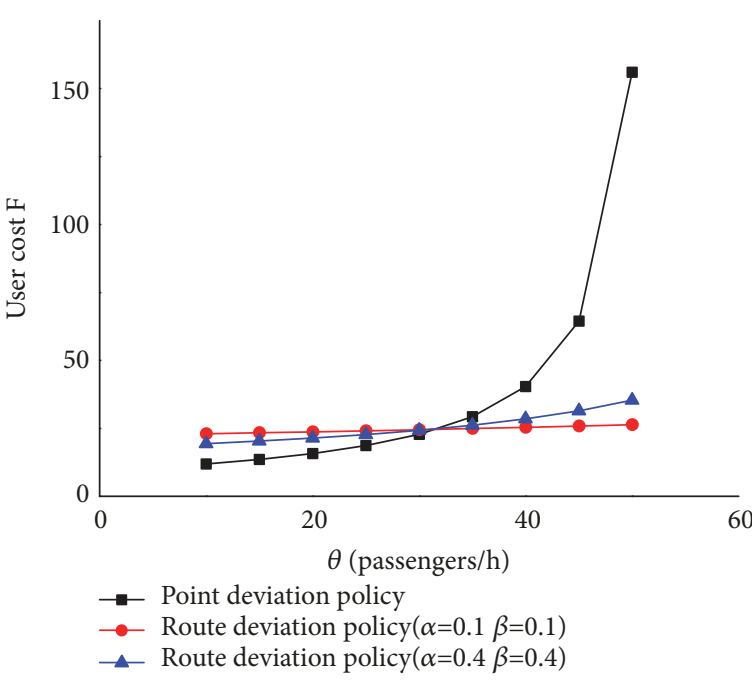

(e) At expected demand ( $W=1.5$ mile $)$

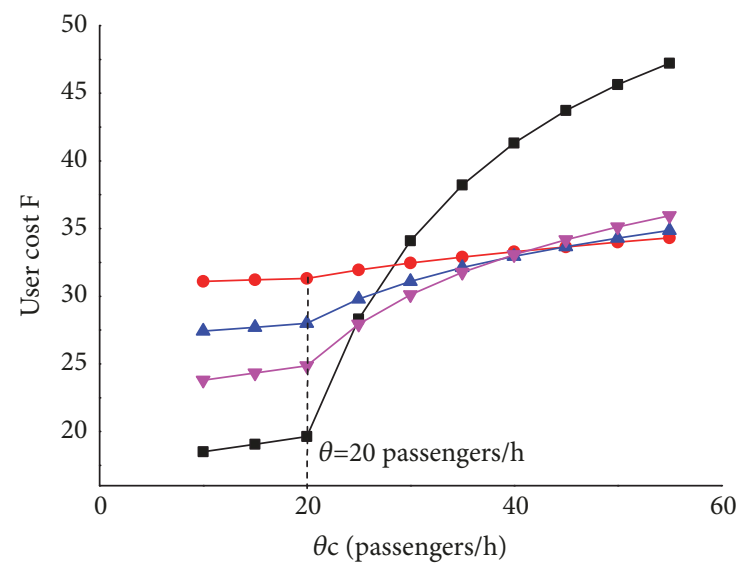

- Point deviation policy

$\multimap$ Route deviation policy $(\alpha=0.1 \beta=0.1)$

$\_$Route deviation policy $(\alpha=0.4 \beta=0.4)$

$\checkmark$ Route deviation policy $(\alpha=0.7 \beta=0.7)$

(b) At unexpected demand $\left(\omega_{K}=3, \omega_{A}=3, \omega_{R}=1\right)$

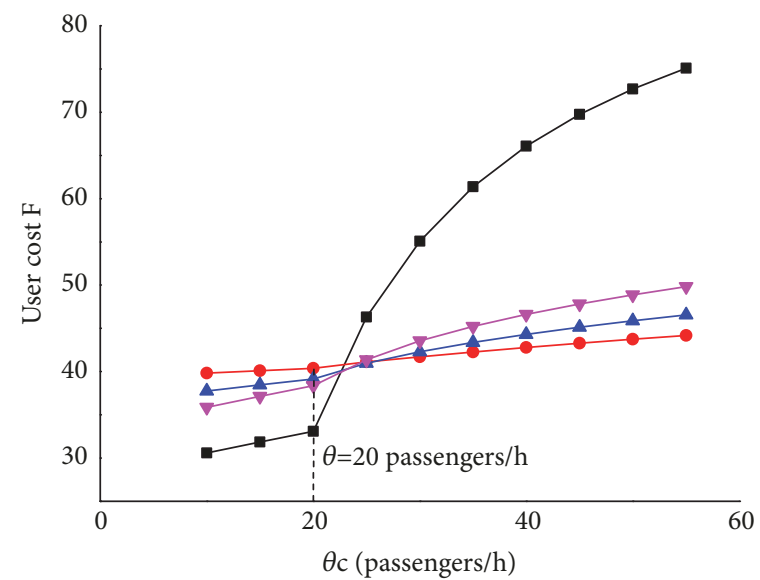

- Point deviation policy

$\rightarrow$ Route deviation $\operatorname{policy}(\alpha=0.1 \beta=0.1)$

$\_$Route deviation policy $(\alpha=0.4 \beta=0.4)$

$\neg-$ Route deviation policy $(\alpha=0.7 \beta=0.7)$

(d) At unexpected demand ( $L=8$ mile)

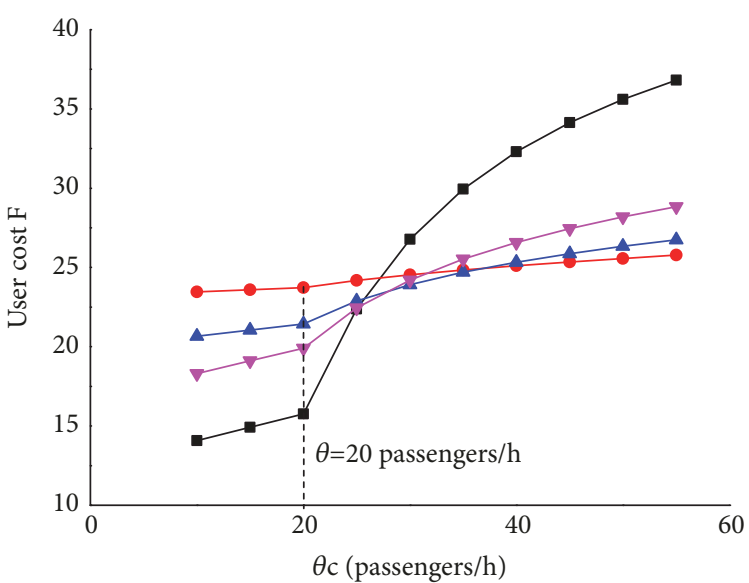

Point deviation policy

$\longrightarrow$ Route deviation policy $(\alpha=0.1 \quad \beta=0.1)$

$\_$Route deviation $\operatorname{policy}(\alpha=0.4 \beta=0.4)$

$\neg$ Route deviation policy $(\alpha=0.7 \beta=0.7)$

(f) At unexpected demand ( $W=1.5$ mile)

Figure 6: Continued. 


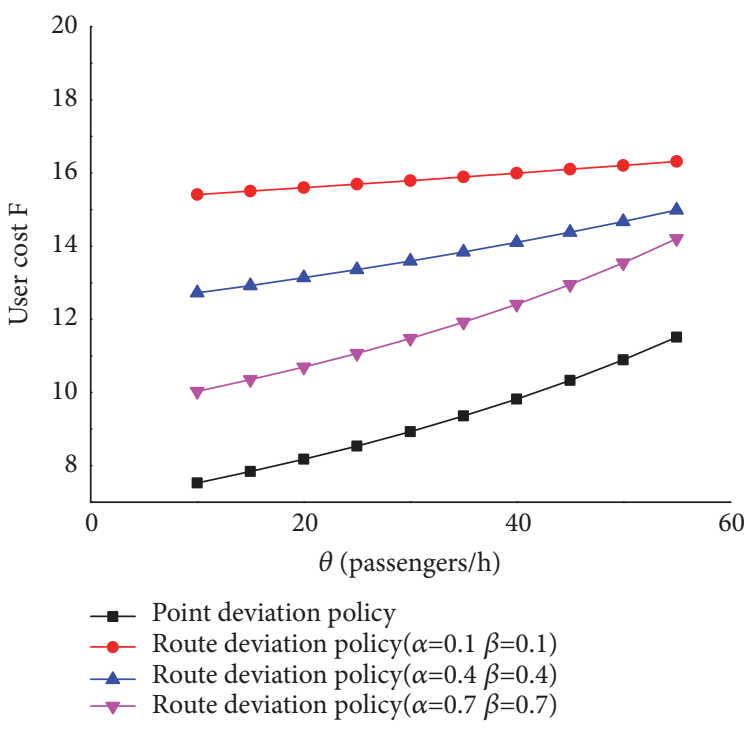

(g) At expected demand $(M=2)$

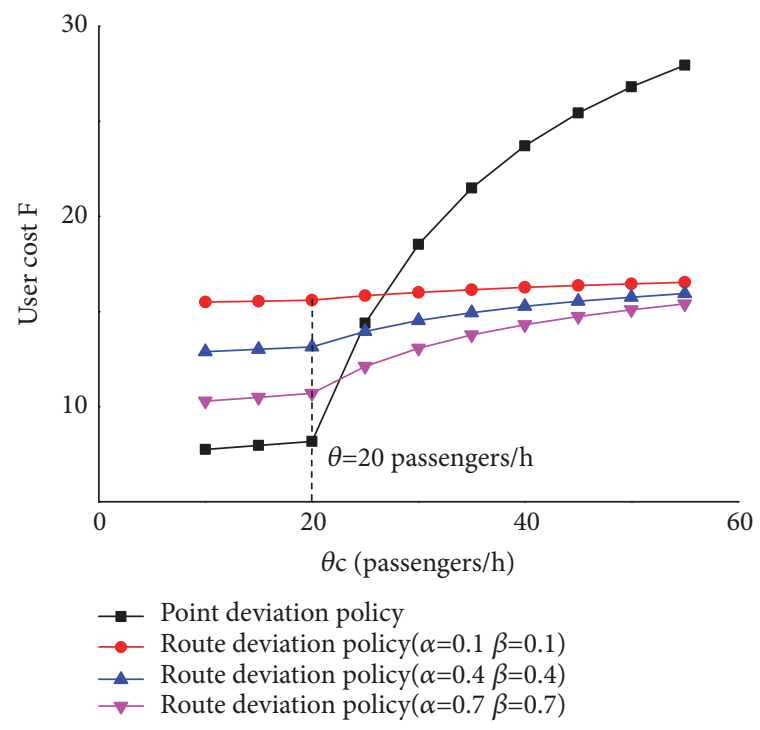

(h) At unexpected demand $(M=2)$

FIGURE 6: Sensitivity analysis for different parameters.

The results suggest that point deviation policy is preferable at low-demand levels and that route deviation policy is better at low-to-moderate demand levels. When the proportion of on-demand requests is considerably large, route deviation is not preferred even at relatively high demand. Route deviation policy is considered to be more reliable because the rejected curb-to-curb customers can still get access to transit with a shorter walking distance. In contrast, point deviation policy might not be suitable when the demand in the service area fluctuates significantly.

In practical use, point deviation service is more complex to implement than route deviation service because it requires a more sophisticated navigation and scheduling system. In addition, the travel time between checkpoints is more difficult to determine in point deviation service since the path might change for each ride. These difficulties explain why this promising operating policy has been applied by only a small percentage of transit agencies compared to route deviation policy. Therefore, an automated dispatch system with high navigation accuracy and an imbedded routing algorithm are needed to promote the widespread use of point deviation service. For future study, we plan to develop simulation models to reproduce the operation of these two competing service types more accurately considering the vehicle capacity and passengers' travel mode choice. Another possible research direction is to compare other kinds of flexible transit policies and define their application conditions.

\section{Data Availability}

The data used to support the findings of this study are available from the corresponding author upon request.

\section{Disclosure}

This paper is an extended version of paper "Flexible Transit Services Choosing between Route Deviation and Point Deviation Policy" presented in 2018 TRB Annual Meeting.

\section{Conflicts of Interest}

The authors declare that they have no conflicts of interest.

\section{Acknowledgments}

The research reported in this paper was supported by the National Natural Science Foundation of China (Grant no. 61573098).

\section{References}

[1] D. Gupta, H. W. Chen, L. A. Miller, and F. Surya, "Improving the efficiency of demand-responsive paratransit services," Transportation Research Part A: Policy and Practice, vol. 44, no. 4, pp. 201-217, 2010.

[2] J. Becker, R. Teal, and R. Mossige, "Metropolitan transit agency's experience operating general-public demand-responsive transit," Transportation Research Record, no. 2352, pp. 136-145, 2013.

[3] S. R. Fittante and A. Lubin, "Adapting the Swedish service route model to suburban transit in the United States," Transportation Research Record, vol. 2536, pp. 52-59, 2015.

[4] D. Koffman, "Operational Experiences with Flexible Transit Services: A Synthesis of Transit Practice," TCRP Synthesis 53, Washington, Wash, USA, 2004.

[5] J. F. Potts, M. A. Marshall, E. C. Crockett, and J. Washington, "A Guide for Planning and Operating Flexible Public Transportation Services," Tech. Rep. 140, Transportation Research Board, Washington, Wash, USA, 2010. 
[6] N. R. Velaga, J. D. Nelson, S. D. Wright, and J. H. Farrington, "The potential role of Flexible Transport Services in enhancing rural public transport provision," Journal of Public Transportation, vol. 15, no. 1, pp. 111-131, 2012.

[7] J. Yu, X. Lu, S. Pan, and C. Guo, "Traveler willingness to use flexible transit services in China: Case study of Qilu Software Park," Journal of Urban Planning and Development, vol. 143, no. 2, 2017.

[8] C. Frei, M. Hyland, and H. S. Mahmassani, "Flexing service schedules: Assessing the potential for demand-adaptive hybrid transit via a stated preference approach," Transportation Research Part C: Emerging Technologies, vol. 76, pp. 71-89, 2017.

[9] F. Errico, T. G. Crainic, F. Malucelli, and M. Nonato, "A survey on planning semi-flexible transit systems: Methodological issues and a unifying framework," Transportation Research Part C: Emerging Technologies, vol. 36, pp. 324-338, 2013.

[10] Tennessee Department of Transportation (TDOT), City of Gallatin Transit Feasibility Study, Tennessee Department of Transportation (TDOT), 2005, https://www.gallatintn.gov/DocumentCenter/View/1208/Gallatin-Transit-Feasibility-StudyDecember-2005-PDF.

[11] L. Quadrifoglio, R. W. Hall, and M. M. Dessouky, "Performance and design of mobility allowance shuttle transit services: Bounds on the maximum longitudinal velocity," Transportation Science, vol. 40, no. 3, pp. 351-363, 2006.

[12] L. Quadrifoglio, M. M. Dessouky, and K. Palmer, "An insertion heuristic for scheduling mobility allowance shuttle transit (MAST) services," Journal of Scheduling, vol. 10, no. 1, pp. 2540, 2007.

[13] L. Quadrifoglio, M. M. Dessouky, and F. Ordóñez, "Mobility allowance shuttle transit (MAST) services: MIP formulation and strengthening with logic constraints," European Journal of Operational Research, vol. 185, no. 2, pp. 481-494, 2008.

[14] L. Fu, "Planning and design of flex-route transit services," Transportation Research Record, no. 1791, pp. 59-66, 2002.

[15] J. Zhao and M. Dessouky, "Service capacity design problems for mobility allowance shuttle transit systems," Transportation Research Part B: Methodological, vol. 42, no. 2, pp. 135-146, 2008.

[16] S. M. Nourbakhsh and Y. Ouyang, "A structured flexible transit system for low demand areas," Transportation Research Part B: Methodological, vol. 46, no. 1, pp. 204-216, 2012.

[17] F. Qiu, W. Li, and A. Haghani, "A methodology for choosing between fixed-route and flex-route policies for transit services," Journal of Advanced Transportation, vol. 49, no. 3, pp. 496-509, 2015.

[18] F. Qiu, J. Shen, X. Zhang, and C. An, "Demi-flexible operating policies to promote the performance of public transit in lowdemand areas," Transportation Research Part A: Policy and Practice, vol. 80, pp. 215-230, 2015.

[19] H. Yang, C. R. Cherry, R. Zaretzki, M. S. Ryerson, X. Liu, and $\mathrm{Z}$. Fu, "A GIS-based method to identify cost-effective routes for rural deviated fixed route transit," Journal of Advanced Transportation, vol. 50, no. 8, pp. 1770-1784, 2016.

[20] P. W. Chen and Y. M. Nie, "Analysis of an idealized system of demand adaptive paired-line hybrid transit," Transportation Research Part B: Methodological, vol. 102, pp. 38-54, 2017.

[21] F. Qiu, W. Li, and J. Zhang, "A dynamic station strategy to improve the performance of flex-route transit services," Transportation Research Part C: Emerging Technologies, vol. 48, pp. 229-240, 2014.
[22] Y. Zheng, W. Li, and F. Qiu, "A slack arrival strategy to promote flex-route transit services," Transportation Research Part C: Emerging Technologies, vol. 92, pp. 442-455, 2018.

[23] C. F. Daganzo and J. Pilachowski, "Reducing bunching with busto-bus cooperation," Transportation Research Part B: Methodological, vol. 45, no. 1, pp. 267-277, 2011.

[24] L. Quadrifoglio, M. M. Dessouky, and F. Ordóñez, "A simulation study of demand responsive transit system design," Transportation Research Part A: Policy and Practice, vol. 42, no. 4, pp. 718737, 2008.

[25] X. Li and L. Quadrifoglio, "Feeder transit services: Choosing between fixed and demand responsive policy," Transportation Research Part C: Emerging Technologies, vol. 18, no. 5, pp. 770780, 2010.

[26] L. Quadrifoglio and X. Li, "A methodology to derive the critical demand density for designing and operating feeder transit services," Transportation Research Part B: Methodological, vol. 43, no. 10, pp. 922-935, 2009.

[27] M. Wardman, "Public transport values of time," Transport Policy, vol. 11, no. 4, pp. 363-377, 2004. 


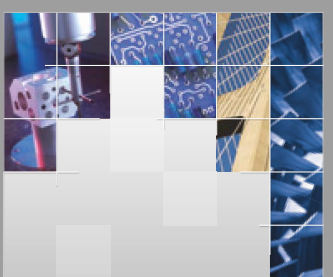

\section{Enfincering}
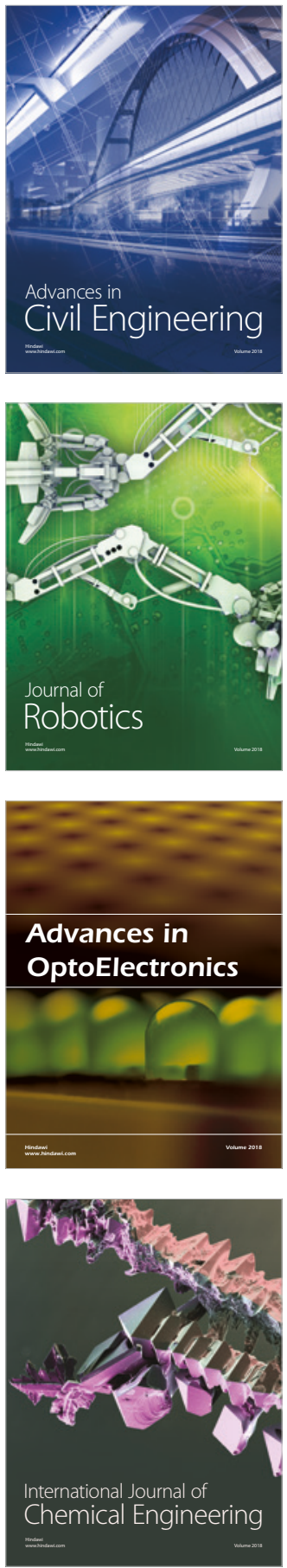

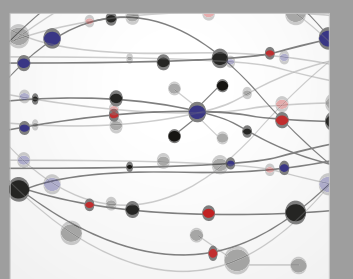

\section{Rotating \\ Machinery}

The Scientific World Journal

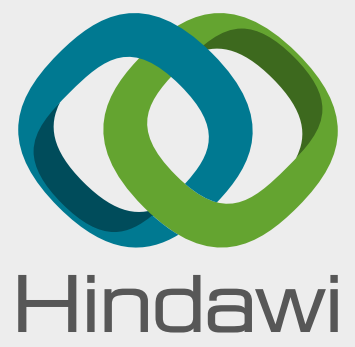

Submit your manuscripts at

www.hindawi.com
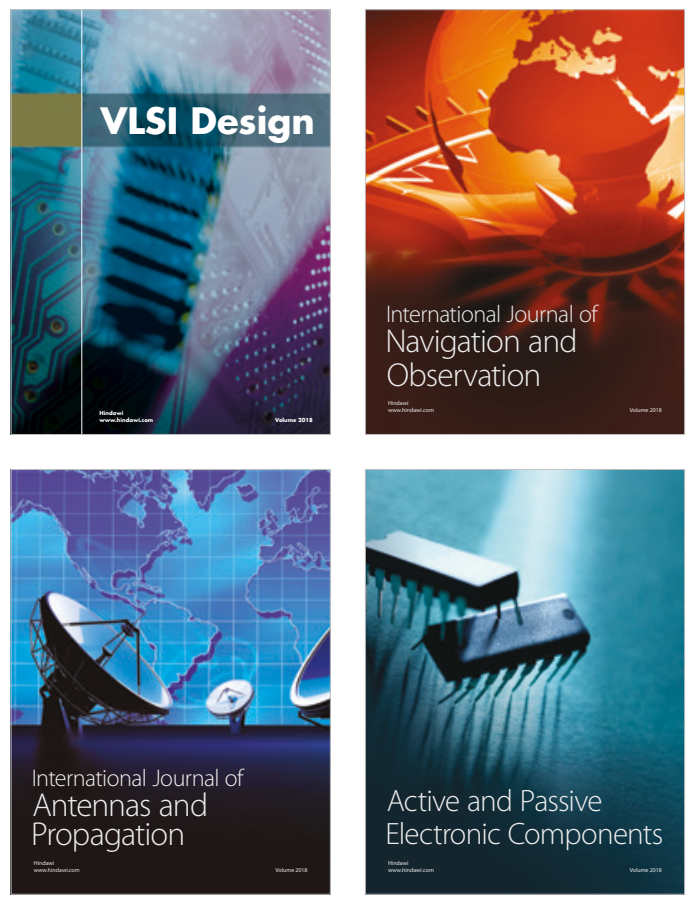
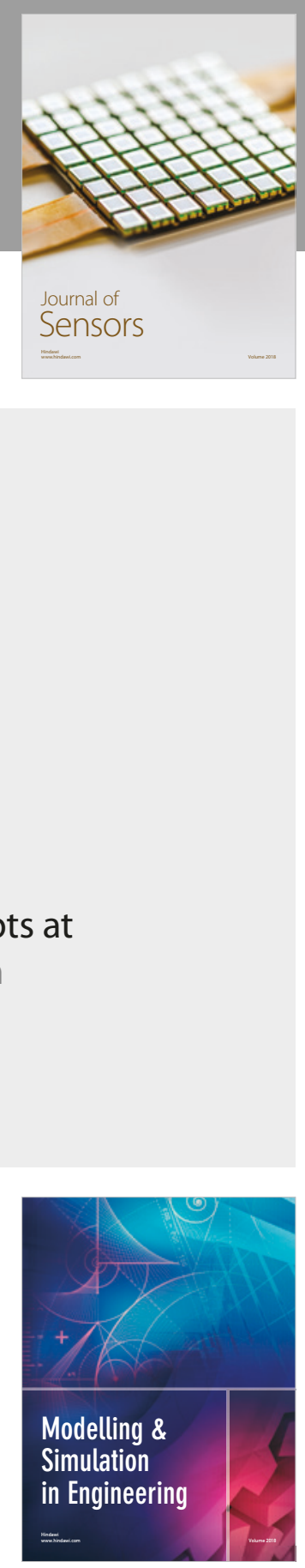

\section{Advances \\ Multimedia}
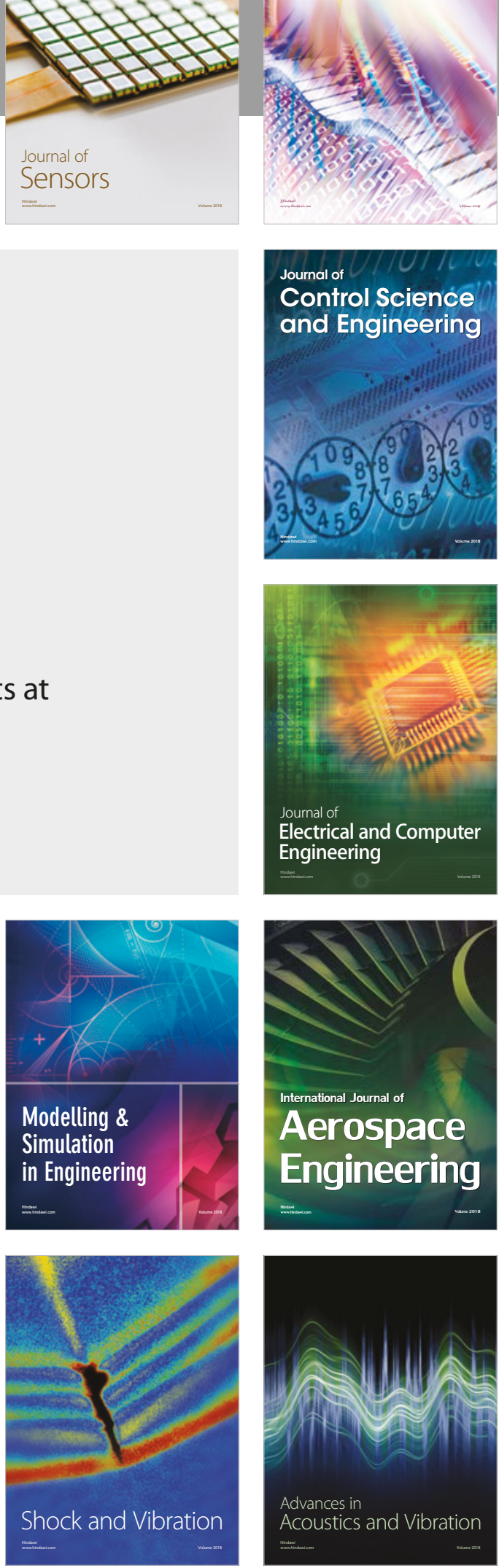